\title{
Identification and management of drug-drug interactions between hydroxychloroquine and long-term treatments in COVID-19 patients
}

\author{
Marin Lahouati ${ }^{1}$, Aurélie Labadie ${ }^{1}$, Julien Nunes ${ }^{1}$, Etienne Meriglier ${ }^{1}$, Aimée Minot ${ }^{1}$, \\ Fabrice Bonnet ${ }^{1}$, and Stéphane Pedeboscq ${ }^{1}$ \\ ${ }^{1}$ Centre Hospitalier Universitaire de Bordeaux
}

May 4, 2020

\begin{abstract}
Due to COVID-19 global pandemic, treatment with HCQ, although controversial, has been widely prescribed. The aim of our study was to identify drug-drug interactions (DDI) between HCQ and long-term treatments of COVID-19 patients, focusing on drugs which can cause or promote QT prolongation. From March 13 to April 14 2020, 61 patients were treated with HCQ in our hospital. More than a half of patients $(62 \%)$ had at least one DDI between HCQ and their long-term treatments. A large number of drug classes were involved, included contraindicated drugs The mean of co-medication contraindicated or not recommended with HCQ per patient was 1.9 CI95\% [1.48 to 2.27]. All contraindicated drugs were discontinued at the beginning of HCQ treatment due to multidisciplinary meetings involving physicians and clinical pharmacists. To our knowledge this is the first study about DDI between HCQ and long-term treatments of COVID-19 patients.
\end{abstract}

\section{INTRODUCTION}

Hydroxychloroquine (HCQ) is a hemi-synthetic drug used for its immunomodulatory activity in a wide range of pathologies, in particular autoimmune and inflammatory diseases since years. The mechanism of action remains unclear but it seems probably multifactorial including immunomodulatory and anti-inflammatory effects. Knowing its effect on the immune system and its safety profile, researchers wondered about its use in other areas, such as viral infections ${ }^{1}$.

Since the beginning of coronavirus disease 2019 (COVID-19) outbreak, several studies report in vitro activity of HCQ on Sars-Cov- $2^{2,3}$, but its clinical efficacy remains controversial at this time, because of the lack of strong in vivodata from randomized studies ${ }^{4}$. However, with the rapid spread of COVID-19 and on the basis of current knowledge, many prescribers have already introduced HCQ-based therapies which became a standard of care in many guidelines ${ }^{5}$.

Safety profile and side effects are well known for patients with autoimmune or inflammatory diseases. As part of COVID-19 pandemic, Yaoet al . report that using HCQ is safe ${ }^{2}$. Indeed, HCQ treatment is generally well tolerated and side effects are usually dose and duration related ${ }^{6}$.

However, adverse effects can occur for short-term treatments and one of the most described side effect could be the risk of cardiovascular disorders, such as QT prolongation ${ }^{7}$.

In the context of COVID-19 pandemic and off-label HCQ treatment, 80 cases of heart disorders associated with HCQ prescription (alone or in combination) were reported on April $24^{\text {th }}$ by the French National Agency for Medicines and Health Products Safety ${ }^{8}$. 
Treatment by drugs known to prolong the QT interval increases the risk of developing arrhythmia during HCQ-treatment. Indeed, the combination of several drugs prolonging the QT interval enhances this risk ${ }^{9}$.

In the largest cohort to date, investigators stated that they discontinued any drug potentially prolonging the QT during HCQ treatment but the number and frequency of treatment at risk of increasing the QT are not specified $^{10}$.

Thus, the aim of our study is to identify drug-drug interactions (DDIs) between HCQ and long-term treatments of COVID-19 patients focusing on drugs that can cause or promote QT prolongation and way to manage them.

\section{PATIENTS AND METHODS}

This work is an observational study on all patients treated with HCQ for COVID-19 infection in medicine care services of the Bordeaux university hospital. Prescriptions and baseline characteristics were analyzed during four weeks.

The analysis of DDIs was focused on cardiovascular side effects, especially QT prolongation or Torsades de Pointe (TdP). For each drug, we assessed the risk of prolonging the QT interval and causing arrhythmia by using two publicly available reference sites: CredibleMeds.org and french-drugs database Thériaque $\mathbb{R}{ }^{11}$.

Thus, these drugs were divided into four broad categories: drugs contraindicated with HCQ (drugs associated with a known risk of QT prolongation or TdP), drugs not recommended with HCQ (drugs associated with a possible risk of QT prolongation), drugs that do not prolong the QT interval per se but can create clinical or biological conditions that facilitate or induce QT-prolongation (bradycardia or hypokalemia) and drugs without impact on QT-prolongation. We also analyzed how these DDIs were managed.

\section{RESULTS}

\section{Patients' characteristics}

From March 13 to April 14 2020, 61 patients were treated with HCQ. Their characteristics are summarized in Table 1. The median age was 72 and $44 \%$ were women. The median number of long term treatment perpatient was 4.

\section{Drugs and QT prolongation:}

Among the admission treatments, a total of 125 different drugs were found, involved in a total of 304 prescription lines. Drugs were splitted into the four broad categories as follows: five drugs (13 prescriptions) were contraindicated with HCQ, 22 drugs (47 prescriptions) were not recommended with HCQ, 7 drugs (24 prescriptions) were at risk of promoting QT prolongation by inducing bradycardia or hypokalemia and 91 drugs (220 prescriptions) had no impact on QT prolongation. Drug classes are reported in Table 2.

\section{DDIs between long-term treatments and HCQ}

A total of 38 patients $(62 \%)$ had a potential increased risk of QT prolongation due to DDIs between their long-term treatments and HCQ.

Among them, 32 patients had at least one co-medication contraindicated or not recommended with HCQ. The mean of co-medication contraindicated or not recommended with HCQ per patient was $1.9 \mathrm{CI}_{95 \%}[1.48$ to 2.27] and 10 patients had 3 or more of these co-medications.

More precisely, 13/32 patients had a contraindicated drug with HCQ. Drugs involved were amiodarone 5/13, escitalopram 5/13, fluconazole $1 / 13$, hydroxyzine $1 / 13$, and levofloxacine $1 / 13$. Moreover, a drug that could create a clinical or biological situation facilitating or inducing QT prolongation (hypokalemia or bradycardia) was also a part of the long term-treatments of $14 / 32$ patients.

The other 6/38 patients only had a co-medication that could induce a clinical situation at risk of QT prolongation. 


\section{Managing DDIs}

All HCQ prescriptions were analyzed by clinical pharmacists. Then multidisciplinary meetings were carried out with physicians in order to evaluate and manage DDIs.

Among the 60 contraindicated and not recommended drugs with HCQ, $32(53 \%)$ were stopped or modified: 25 before starting HCQ treatment and 7 during HCQ treatment. All the contraindicated drugs $(\mathrm{n}=13)$ were discontinued: 9 before initiation of HCQ, 3 on the first day of treatment and 1 two days after HCQ initiation.

The other 28 not recommended drugs $(47 \%)$ were not discontinued during HCQ treatment. Main drugs were PPI (10/28), loop diuretic (5/28) and inhaled-bronchodilator $(4 / 28)$.

\section{DISCUSSION}

Due to SARS-CoV2 global pandemic, treatment with HCQ, although controversial, has been widely prescribed. These prescriptions have been made in an emergency context and lack of open clinical trials at the very beginning of the outbreak. Although the side effects profile of HCQ is well known for decades, the population receiving this medication is different from the one usually treated for auto-immune diseases ${ }^{6}$, so the DDIs encountered could be unusual. We, hereby, analyze the potential DDIs found in this population with a focus on cardiac side effects.

In this study, a large number of patients had risk factors of QT prolongation (age over 65, sex or comorbidities as renal or heart failure $)^{9}$. These patients' characteristics may explain the number of long-term treatments, increasing risk of QT prolongation due to DDIs, especially with HCQ. More than a half of patients had at least one DDIs between their long-term treatments and HCQ. A large number of drug classes were involved, included contraindicated drugs which were discontinued at the beginning of HCQ treatment. However, as some of these drugs have a long elimination half-life (30 hours for escitalopram, up to 100 days for amiodarone ${ }^{11}$ ), a residual risk could persist, even after the drug in question is stopped.

For each not recommended drug, benefit-risk ratio was assessed by physicians and clinical pharmacists. Among the involved drugs, there were also some widespread medications, like PPIs or inhaled-bronchodilators. These drugs were often continued during HCQ treatment, which could be explained by lack of information about their potential impact on the QT interval. Nevertheless these DDIs have to be taken into account during treatment with HCQ especially as COVID-19 can manifest by acute myocardial injury, which could lead to cardiac arrhythmias ${ }^{12}$.

This study shows that a huge number of drugs could interact with HCQ. Consequently, it is of great interest to upstream study the QT prolongation risks due to co-medication in COVID-19 patients treated with this drug. Physicians must be aware of QT prolongation risk when HCQ is associated with other drugs increasing the QT interval per se in the treatment of COVID-19, such as azithromycin or protease inhibitors.

In addition to this well described cardiac toxicity, it is also important to consider DDIs which may lead to other side effects such as hypoglycemia or increasing risk of convulsion ${ }^{13}$. Moreover HCQ is substrate of CYP 450 and is an inhibitor of CYP 2D6 so pharmacokinetics DDIs should be taken into account.

In this context of pandemic, clinical pharmacists should analyze all the prescriptions of COVID-19 patients in order to detect DDIs and discuss with physicians the opportunity of stopping a medication, adapting a posology or in some cases, pursuing the involved drug.

To our knowledge this is the first study about DDIs between HCQ and long-term treatments of COVID-19 patients.

Other studies, including clinical and pharmacokinetic parameters, need to be carried out subsequently to measure impact of DDIs among COVID-19 patients.

\section{ACKNOWLEDGEMENTS}

ML, AL, JN, AM, FB and SP were involved in the conception of the study. 
ML, AL and EM contributed to the study design including data collection and analysis. All authors contributed to manuscript draft and revisions.

\section{DECLARATION OF INTEREST}

The authors have no conflicts of interest to declare.

\section{DATA AVAILABILITY STATEMENT}

The data that support the findings of this study are available on request from the corresponding author. The data are not publicly available due to privacy or ethical restrictions.

\section{REFERENCES}

1. Savarino A, Boelaert JR, Cassone A, Majori G, Cauda R. Antiviral effects of chloroquine Effects of chloroquine on viral infections : an old drug against today 's diseases ? Lancet. 2003;3(November):722727. doi:10.1016/S1473-3099(03)00806-5

2. Yao X, Ye F, Zhang M, et al. In Vitro Antiviral Activity and Projection of Optimized Dosing Design of Hydroxychloroquine for the Treatment of Severe Acute Respiratory Syndrome Main point : Hydroxychloroquine was found to be more potent than chloroquine at inhibiting SARS-CoV-2 in vit. Clin Infect Dis. 2020;2:1-25.

3. Wang M, Cao R, Zhang L, et al. Remdesivir and chloroquine effectively inhibit the recently emerged novel coronavirus (2019-nCoV) in vitro. Cell Res. 2020;30(3):269-271. doi:10.1038/s41422-020-0282-0

4. He F, Deng Y, Li W. Coronavirus disease 2019: What we know? J Med Virol. 2020;2019:0-2. doi:10.1002/jmv.25766

5. Haut Conseil de la Sante Publique. Avis relatif aux recommandations therapeutiques dans la prise en charge du COVID-19.https://www.hcsp.fr/explore.cgi/avisrapportsdomaine? clefr=785. Published March 2020. Accessed March 24, 2020.

6. McGhie TK, Harvey P, Su J, Anderson N, Tomlinson G, Touma Z. Electrocardiogram abnormalities related to anti-malarials in systemic lupus erythematosus. Clin Exp Rheumatol. 2018;36(4):545-551.

7. White NJ. Cardiotoxicity of antimalarial drugs. Lancet Infect Dis. 2007;7(8):549-558. doi:10.1016/S14733099(07)70187-1

8. Agence Nationale de Securite des Medicaments et des Produits de Sante. Bilan des effets indesirables valides dans la base nationale de pharmacovigilance concernant les patients pris en charge dans le contexte d'une infection a COVID-19 et mesusage. Published April 2020. Accessed April 27, 2020.

9. Societe Francaise de Cardiologie. Preconisations cardiologiques lors de l'utilisation d'un traitement pouvant entrainer des troubles conductifs ou un allongement de l'intervalle QT dans le COVID-19. Published April 2020. Accessed April 15, 2020.

10. Gautret P, Lagier J-C, Parola P, et al. Clinical and microbiological effect of a combination of hydroxychloroquine and azithromycin in 80 COVID-19 patients with at least a six-day follow up: A pilot observational study. Travel Med Infect Dis. 2020.

11. Husson MC. Theriaque(r) : base de donnees independante sur le medicament, outil de bon usage pour les professionnels de sante. Ann Pharm Fr. 2008;66(5-6):268-277. doi:10.1016/j.pharma.2008.07.009

12. Hendren NS, Drazner MH, Bozkurt B, Cooper LT. Description and Proposed Management of the Acute COVID-19 Cardiovascular Syndrome. Circulation. 2020. doi:10.1161/CIRCULATIONAHA.120.047349

13. Malcangi G, Fraticelli P, Palmieri C, Cappelli M, Danieli MG. Hydroxychloroquine-induced seizure in a patient with systemic lupus erythematosus. Rheumatol Int. 2000;20(1):31-33. doi:10.1007/s002960000066

\section{Hosted file}

Tables . docx available at https://authorea.com/users/317415/articles/447523-identification-andmanagement-of-drug-drug-interactions-between-hydroxychloroquine-and-long-term-treatmentsin-covid-19-patients 